\title{
UN MODELO DE COEVOLUCIÓN ENTRE LA GOBERNANZA SOCIAL Y LA MODERNIZACIÓN ECONÓMICA
}

\author{
Manuela Armas Carrillo* \\ Departamento de Finanzas y Contaduría, Universidad de las Américas Puebla \\ (Recibido 16 agosto de 2013, aceptado 18 de marzo de 2014)
}

\section{Resumen}

En este artículo se desarrolla un modelo que trata de explicar la coevolución entre la gobernanza social y la modernización económica. Partiendo de una economía tradicional (transacciones relacionales y grupos con un alto grado de cohesión social) se llega a una economía moderna (transacciones en mercados anónimos y un insignificante grado de cohesión social). En la transición, la disminución en la cohesión social puede causar que la cooperación colapse si la economía cruza un umbral de complejidad (comercio a distancia) antes de que surjan las sanciones de terceros. En este sentido se dice que las instituciones son cruciales para que el mercado se desarrolle y la cooperación se generalice. El modelo hace uso de una red endógena, en la que los agentes interactúan a través de un juego evolutivo espacial.

\section{Abstract}

This article develops a model that treats to explain the coevolution between the social governance and the economic modernization. On the basis of a traditional economy -relational transactions and, groups with a high grade of social cohesion- a modern economy is reached- transactions in anonymous markets and an insignificant grade of social cohesion. In the transition, the decrease in the social cohesion may cause that this cooperation collapses if the economy crosses a threshold of complexity (distance trade) before sanctions to third parties emerges. In this sense it is said that institutions are crucial for market development and the generalization of cooperation. The model uses an endogenous network, in which the agents interact through a space evolution game.

Clasificación JEL: M14, M13, G3.

Palabras clave: Cambio cultural, Coevolución, Gobierno económico.

\footnotetext{
* Universidad de las Américas Puebla, Escuela de Negocios y Economía - Sta. Catarina Mártir, Cholula, Puebla, México. C.P. 72810 Tel. +52 (222) 22924 60. Correo electrónico: manuela.armas@udlap.mx
} 


\section{Introducción}

El presente trabajo de investigación tiene como objetivo explicar el desempeño de una sociedad en su proceso de modernización económica. Una economía tradicional se caracteriza por transacciones relacionales y grupos con un alto grado de cohesión social que dan lugar a creencias e intereses compartidos. En cambio en una economía moderna los mercados son anónimos y existe un grado de cohesión social insignificante, es decir la economía se caracteriza por interacciones a distancia.

La hipótesis de investigación que se propone en el artículo es la siguiente. En una economía tradicional se preserva la cooperación de la comunidad gracias a la elevada cohesión social, pero se retarda el desarrollo del comercio debido al casi inexistente comercio a distancia. Cuando se comienzan a incrementar las interacciones a distancia se desarrolla el comercio pero también se pierde la cohesión de la comunidad y con ella la cooperación. Para evitar que la cooperación colapse deben surgir instituciones de terceros en la sociedad que refuercen el comportamiento cooperativo, lo que permite disminuir el oportunismo en los intercambios comerciales y hacer posible el despegue de la economía.

Para probar teóricamente la hipótesis, aquí se modela el proceso de construcción de una economía de mercado (transacciones a distancia). Se parte de una situación en donde los actores realizan exclusivamente transacciones relacionales en un contexto de dilema del prisionero. Los jugadores aprenden a través del tiempo que estrategia les conviene, por lo que imitan la estrategia de comportamiento del compañero de juego que obtuvo un mejor resultado. Paulatinamente, aquellos actores con poder económico comienzan a establecer transacciones a distancia sacrificando relaciones cercanas, si lo consideran económicamente beneficioso. Eventualmente surgen elites económicas que comienzan a sancionar el oportunismo en sus relaciones, promoviendo de esta manera que el mercado se desarrolle y la cooperación se generalice. Para modelar lo anterior se propone un juego evolutivo espacial en el que los individuos se ubican en los nodos de una red endógena y los lazos de la red definen las conexiones que existen entre ellos.

Lo que resta del artículo se estructura de la siguiente forma. En la segunda sección se revisa la literatura que motivó la hipótesis de investigación. En la tercera sección se comenta la literatura relacionada con el análisis de este artículo. En la cuarta sección se comentan los juegos evolutivos espaciales. En la quinta sección se presenta el modelo de investigación. En la sexta sección se discuten los resultados de las simulaciones del modelo de investigación. Por último, se presentan las conclusiones.

\section{Motivación}

El estudio de Ensminger (1992) sobre la tribu Orma de Kenia es apropiado para describir el proceso coevolutivo que nos preocupa. Su estudio documenta el proceso del desarrollo de mercado que tuvo lugar entre la Orma Galole pastoral y la sociedad Orma moderna. Ensminger traza los orígenes Orma en Kenia a las migraciones de Etiopía que tuvieron lugar en el temprano s. XVI. Desde entonces y hasta la parte temprana del s. XX, los Ormas fueron principalmente pastores, con una producción de subsistencia y una economía basada en el ganado y la agricultura. La organización en la tribu era muy cohesionada, con 
una cooperación cerrada y patrones de consumo uniformes entre los miembros de la tribu.

Durante este periodo el comercio a distancia (hacia afuera de la tribu) era casi inexistente. Los Ormas tenían una reputación de ser hostiles con individuos ajenos a la tribu, por lo que las caravanas y comerciantes árabes evitaban el territorio Orma. Ensminger nota que la "clausura del territorio Orma ciertamente retardó el desarrollo del comercio pero también ayudó a preservar la cooperación en la tribu y la autoridad del jefe.

A finales del s. XIX la mayoría de África fue colonizada por el Imperio Británico. Después, en el temprano s. XX, los Ormas comenzaron gradualmente a comerciar con otros grupos y tribus. Ensminger atribuye esta apertura a un número de causas. Por un lado, guerras devastadoras con los Masáis y Somalíes, en la segunda mitad del s. XIX, resultaron en la casi aniquilación de los Ormas y crearon un periodo de inseguridad que estresó su organización social, política y económica. Por el otro lado, a inicios del s. XX, un número de desarrollos disminuyeron los costos de transacción del comercio. Todo esto podría interpretarse como un proceso de modernización que Ensminger define como (a) la conversión al Islam que permitió integrar instituciones para gobernar el comercio, en particular el código de honor Islámico y el sistema de crédito Islámico comenda; (b) la estandarización de los pesos y medidas por la injerencia del gobierno colonial Británico; (c) el desarrollo de comunicaciones y transporte en la forma de carreteras, camiones motorizados y telegramas; (d) el establecimiento de una burocracia administrativa para organizar el comercio.

Ensminger describe cómo al incrementarse las interacciones económicas con forasteros y darse una mayor diversidad económica se produjo un fallo de la comunidad y el fracaso de la cooperación y acción colectiva entre los Ormas. Esto precipitó un cambio sustantivo en la forma de la autoridad gubernamental, al pasar de un consejo de ancianos al estado nación de la Kenia moderna. Entre los cambios institucionales resalta el incremento en la autoridad del líder designado para gobernar (un servidor civil del estado) a expensas del consejo de ancianos. En este contexto se hizo común que el gobernante usara la fuerza (policía y cortes) en la administración de los deberes; lo cual representaba una desviación considerable de las prácticas previas.

En la etapa tradicional, muchos grupos étnicos no consideraban como legítimo el uso de la fuerza por el estado en contra de sus miembros. En esa época los asuntos que involucraban la sanción de su propia gente se resolvían a través del uso de consejos de ancianos u otras instituciones ancestrales. Así, en la etapa moderna el gobernante era un hombre Orma joven y educado, y sus deberes eran ejecutados de manera diferente al líder previo, el jefe Orma anciano y analfabeta. Si bien los ancianos no aprobaban del todo lo que el gobernante joven estaba haciendo, ellos apoyaban en gran medida la transición a una creciente autoridad del estado y el uso de la fuerza por el gobernante en contra de su propia gente. Esto significaba que el cambio lejos de ser un cambio exógeno impuesto a los Ormas, reflejaba una necesidad percibida -por al menos algún segmento de la población- de cambiar en las instituciones locales. Ensminger afirma que una de las últimas crisis que llevaron a este cambio fue la necesidad de hacer valer derechos de propiedad nuevos y la incapacidad de los ancianos para lograrlo sin instituciones de terceros, tales como el estado. 
El estudio de Ensminger motiva la hipótesis de investigación del presente trabajo: cuando la organización en una sociedad es altamente cohesionada y el comercio a distancia es casi inexistente se preserva la cooperación de la comunidad pero se retarda el desarrollo del comercio. Así mismo, cuando estas comunidades comienzan a incrementar las interacciones económicas con forasteros se desarrolla el comercio pero también se produce un fallo en la cohesión de la comunidad y un fracaso en la cooperación y acciones colectivas emprendidas por los miembros de la comunidad. Para evitar que la cooperación colapse deben surgir instituciones de terceros en la sociedad que refuercen el comportamiento cooperativo, lo que permite disminuir el oportunismo en los intercambios comerciales y hacer posible el despegue de la economía.

\section{Revisión de la Literatura}

El análisis de este artículo combina un conjunto de planteamientos de la literatura socioeconómica que se sintetizan a continuación.

La teoría de la acción colectiva. Existen dos leyes o aforismos, que de una manera evocativa, resumen la forma de operar de una economía. La primera ley es que "algunas veces, cuando cada individuo considera únicamente sus intereses, emerge automáticamente un resultado colectivamente racional" -la famosa mano invisible que coordina los esfuerzos de todos los individuos involucrados y asegura un resultado socialmente eficiente en el sentido de Pareto (Smith, A., 1976). La segunda ley es que "algunas veces, la primera ley no se cumple: sin importar qué tan inteligentemente los individuos persiguen su propio interés, ningún resultado socialmente racional puede emerger espontáneamente" -por lo que se requiere de una mano visible que guíe o de una institución que ayude a producir resultados colectivamente eficientes (Olson, 1965). Se puede reescribir esta segunda ley de la siguiente forma: "la racionalidad individual no es suficiente para la racionalidad colectiva. Cuando existe la expectativa generalizada entre los individuos de que el accionar de los demás no será cooperativo, se incentiva a cada uno de ellos a actuar de manera oportunista (Chamberlin, 1974; Granovetter, 1978; Marsden, 1981; McAdam, 1983; Runge, 1984).

Los procesos de cambio institucional a partir de los movimientos sociales. Aunque las élites en el poder se vean beneficiadas con la presencia de instituciones extractivas o ineficientes, y tengan claros incentivos en sostenerlas, el problema sigue siendo un problema de acción colectiva debido a que las mayorías desfavorecidas se encuentran fragmentadas y son incapaces de llevar a cabo acuerdos cooperativos; situación que hace muy difícil impulsar reformas que sean más conducentes a la equidad y el crecimiento. El problema de acción colectiva es producto, en gran medida, del contexto cultural e histórico de la sociedad en consideración. Las expectativas de los individuos sobre el comportamiento de las demás personas es sumamente importante para definir la posibilidad de que los movimientos sociales sean cohesionados y tengan la fuerza suficiente para inducir las reformas institucionales requeridas para el desarrollo (Geddes, 1999; McCarthy y Zald, 1977; O'Donnell y Schmitter, 1986; Tilly, 2004).

La naturaleza de las elites. En el planteamiento neoclásico el problema de instituciones "ineficientes se suele reducir a elaborar un diseño institucional óptimo en "el que se "fijan" los incentivos adecuados para desincentivar 
el oportunismo y con ello alentar la productividad, sin embargo, la creación de instituciones "eficientes" no es simplemente un asunto técnico en el que los cambios se dan por decreto. Teóricamente las elites podrían diseñar instituciones democráticas, pero el funcionamiento de éstas se encuentra determinado por la perspectiva de emancipación de la cultura. Una constitución bien escrita no basta para hacerla trabajar. Los derechos formales no pueden ser creados por las elites si no es que existe una demanda por ellos por parte de la ciudadanía (Eckstein, 1988; Elkins, 2000; Iversen, 1994).

La relación entre desarrollo socioeconómico, cambio cultural y democratización. Diversos investigadores interesados en el desarrollo y cambio de las sociedades humanas plantean que existen tres trayectorias mayores de cambio social, siendo la más básica el desarrollo socioeconómico. El segundo proceso se produce con un cambio de los valores de la gente hacia una mayor emancipación. Un tercer proceso involucra a las instituciones políticas de una sociedad y en particular de aquellas que conducen hacia una mayor democracia. La evidencia apunta a que el desarrollo socioeconómico, el cambio cultural y el cambio institucional tienden a manifestarse de manera conjunta (Blomkvist, 2003; Estes, 2000; Flanagan e Inglehart, 1987; Kurzman, 1998).

Sociomática (Castañeda, 2009). En una sociedad, los individuos interactúan en las arenas superficiales (económica, política y social) a partir de una estructura sociocultural, por lo que a pesar de que su comportamiento sea muy sencillo, el resultado de su interacción da lugar a fenómenos relativamente elaborados o patrones emergentes (por ejemplo, las instituciones y la cooperación colectiva). La retroalimentación se manifiesta con la presencia de relaciones bidireccionales agente $\leftrightarrow$ agente o agente $\leftrightarrow$ entorno, lo que da lugar no sólo a los patrones emergentes, sino también a la coevolución entre variables.

La coevolución entre variables se da a través de un proceso simultáneo (coevolución sincrónica) y un proceso que se desarrolla a través del tiempo (coevolución diacrónica). En cada periodo existe una relación simultánea entre las diferentes arenas superficiales debido a la inserción social de la toma de decisiones. Es decir, en cada periodo se inicia una nueva ronda coevolutiva en la que las transformaciones en las arenas superficiales se producen a partir de una estructura sociocultural.

Paralelamente, tiene lugar un proceso que se desarrolla a través del tiempo entre las arenas superficiales y la estructura sociocultural o gobierno social. Es decir, una vez que una fractura profunda rompe el status quo, se inicia una nueva ronda coevolutiva en la que las transformaciones en las arenas superficiales van a la par de modificaciones en el gobierno social. Dado que el gobierno social y el "conocimiento convencional" de las arenas superficiales sintetizan la herencia histórica de la sociedad, cualquier modificación en las instituciones del ámbito socioeconómico se codifica como parte de la herencia colectiva de las futuras generaciones de la comunidad. 


\section{Juegos evolutivos espaciales}

Para formular y verificar la hipótesis de investigación se propone un modelo de co-evolución de la gobernanza social y económica. Se trata de un modelo basado en agentes que hace uso de una red dinámica o endógena. En las redes fijas o exógenas, el comportamiento agregado (M) es función de las decisiones de agentes autónomos (m) que, a su vez, se ven condicionados por el gobierno social heredado (M'). Implícitamente se habla de una relación causal macro $\left(\mathrm{M}^{\prime}\right) \rightarrow$ micro $(\mathrm{m}) \rightarrow$ macro $(\mathrm{M})$, en donde los gobiernos o estructuras sociales (M, M') no son necesariamente iguales (Castañeda, 2009). Por otro lado, en las redes endógenas se explica cómo los comportamientos colectivos inciden a su vez en la toma-de-decisión del agente mediante modificaciones en la topología social y en los "elementos culturales" de una comunidad (M' $\rightarrow \mathrm{m} \rightarrow \mathrm{M} \rightarrow \mathrm{m}$ ' $\rightarrow \cdots)$.

El modelo de investigación que aquí se elabora propone un juego evolutivo espacial en el que los individuos se ubican en los nodos de una red endógena y los lazos de la red definen las conexiones que existen entre ellos. Paulatinamente, la red se va transformando cuando se van reconectando los lazos originales (transacciones relacionales) de tal forma que el individuo que promueve la reconexión termina conectado ahora con una persona afuera de su vecindario (transacciones anónimas o a distancia). De esta manera, los individuos se involucran en cada periodo en un juego del dilema del prisionero con múltiples jugadores. Los jugadores aprenden a través del tiempo que estrategia les conviene, por lo que imitan la estrategia de comportamiento del compañero de juego que obtuvo un mejor resultado.

El que el juego se lleve a cabo primero entre vecinos cercanos, luego con vecinos cercanos y personas distantes, y finalmente entre personas distantes hace factible que surjan procesos emergentes diferentes en relación a la frecuencia relativa de la cooperación y el oportunismo. En un juego evolutivo convencional, en el que no existe una estructura social y por lo tanto el emparejamiento de los jugadores es aleatorio, la selección de una estrategia depende exclusivamente de los beneficios relativos y la frecuencia en que ésta se observa en la población. En cambio, los juegos evolutivos espaciales permiten introducir en el esquema del análisis la importancia de la topología, y por lo tanto del contexto social para describir como un comportamiento particular se propaga en la población.

\section{El modelo}

En "Social Embeddedness and Economic Governance: A Small World Approach" (Kali, 2003), se desarrolla un marco teórico para entender la transformación de la cohesión social conforme una economía se desarrolla. En este esquema la transición de una economía tradicional a una moderna se asocia a un incremento en la probabilidad de interactuar con individuos afuera de un vecindario relacional estrecho. El marco teórico del mundo pequeño de Watts y Strogatz (1998) permite usar esta probabilidad para transitar esta economía de grupos con un alto grado de cohesión social, a una de mercados anónimos con un grado de cohesión social reducido.

Kali nota que las transacciones relacionales facilitan la cooperación pero las transacciones a distancia facilitan, el compartir la información. La modernización causa que las transacciones relacionales se desvanezcan lo que produce un debilitamiento de la cohesión social y por ende, una reducción en 
la habilidad para gobernar las transacciones de tal forma que la cooperación puede llegar a colapsar totalmente.

Kali considera que esto sugiere un rol crucial para la intermediación institucional una vez que el nivel de complejidad en la economía (transacciones a distancia) ha cruzado un cierto umbral. El autor afirma que tanto las instituciones que hacen valer la ley, como las que informan, se vuelven más valiosas conforme la economía se vuelve cada vez más compleja. Del marco teórico de Kali se derivan oportunidades de investigación:

- En su modelo el proceso de modernización de la economía es totalmente exógeno. Consiste en establecer relaciones a distancia con una probabilidad $p$, de tal forma que conforme $p$ aumenta se incrementa la modernización."

- El autor encuentra que para un cierto nivel $p>p^{*}$ (umbral de complejidad), la economía empieza a tener problemas ya que pierde los beneficios de la cohesión en las transacciones relacionales y sin embargo no se pueden materializar buenas transacciones en las relaciones a distancia. Se habla entonces de un "cuello institucional", por lo que se sugiere que se deben crear instituciones de "arriba-abajo" de tal forma que las transacciones de mercado no sean sujetos del oportunismo.

Aunque Kali habla de co-evolución en su planteamiento ésta no existe ya que no hay procesos de retroalimentación, además de que el autor introduce exógenamente a las instituciones sin explicar por qué pueden o no aparecer.

En el presente trabajo se modela el proceso de construcción de una economía de mercado (transacciones a distancia). Partiendo de una situación en donde cerca de la mitad de la población se comporta cooperativamente y en donde la economía es tradicional, los actores realizan exclusivamente transacciones relacionales en un contexto de dilema del prisionero. Con el paso del tiempo los jugadores van aprendiendo cuál estrategia de comportamiento les conviene más al observar la estrategia de comportamiento del compañero de juego que obtuvo un resultado mayor.

Se supone que el comercio a distancia reditúa beneficios adicionales por la especialización, a pesar de que es necesario invertir en bienes de capital para que el mismo se lleve a cabo. De esta manera, con una probabilidad que está en función del beneficio neto del comercio a distancia, aquellos individuos que han acumulado un cierto nivel de riqueza comienzan a establecer relaciones a distancia sacrificando relaciones cercanas.

En el modelo se hace referencia a la creación de instituciones o sanción entre terceros cuando el porcentaje de individuos con un cierto poder económico alcanza cierto umbral, lo que permite sancionar el oportunismo en las relaciones de estas personas; de esta manera las instituciones en la economía surgen de "abajo-arriba".

\subsection{Dinámicas de interacción}

La topología de la economía se conforma con $N$ individuos que constituyen los nodos o vértices de una red en forma de anillo de una dimensión. Cada individuo $i$ está directamente conectado con lazos (o bordes) sin dirección, con otros $k$ en el anillo. Para describir una economía tradicional se supone que las conexiones son con los $k$ vecinos más cercanos. Así cada vértice es de grado $k$. Se supone que $N \gg k \gg \ln (N)$, por lo que la primera desigualdad asegura que 
la gráfica sea escasa, mientras que la segunda evita que existan nodos que no estén conectados.

Dos estadísticos de la red relacional son de especial interés. El primero es la longitud del camino característica, $A P L(N, k)$, que es el mínimo promedio de lazos (del conjunto de lazos) que deben ser recorridos con objeto de alcanzar el vértice $j$ desde el vértice $i$; es decir, la distancia típica entre cada vértice y cada otro vértice, $d(i, j)$. El segundo estadístico es el coeficiente de clustering, $C C(N, k)$, que describe en qué extensión los vértices conectados a cualquier vértice $v$, están conectados entre ellos.

Estos dos estadísticos tienen un significado intuitivo para la economía en consideración. La APL describe que tan relevantes son las transacciones a distancia viz-a-viz las relacionales. El CC refleja el grado de cohesión de la comunidad; es decir, el coeficiente mide el grado de exclusividad de un círculo de conocidos típico. Para modelar el proceso de modernización de una economía se analiza la manera en que se va transformando la red relacional en una red aleatoria, sin alterar el número de vértices o lazos en la red. Con este propósito se toma el enrejado de anillo ordenado de una dimensión, en el cual cada vértice tiene precisamente $k$ vecinos ( $k / 2$ en cada lado) y entonces se reconectan los bordes utilizando el siguiente algoritmo.

Se escoge aleatoriamente un vértice $(i)$ una vez que el nodo acumula una riqueza al menos igual a un cierto umbral $\Omega^{*}$ y el borde que lo conecta a su vecino más cercano $(i+1)$ en el sentido del reloj. Con una probabilidad $p \in(0,1)$ se reconecta este borde de tal forma que $i$ se conecta a otro vértice $j$ el cual también se escoge uniformemente de manera aleatoria sobre el anillo entero, pero sin duplicar lazos. De otra manera se deja el borde en su lugar.

Se repite este procedimiento moviéndose en el anillo en el sentido del reloj hasta que se completa una vuelta. Luego se continúa este proceso de reconexión pero ahora considerando, para cada vértice, el borde que lo conecta a su segundo vecino más cercano; y así sucesivamente, procediendo hacia vecinos más distantes después de cada vuelta, hasta que cada borde en el enrejado original ha sido considerado una vez. Procedimiento que se repite en cada periodo de tiempo. Como se supone una economía sin crecimiento poblacional -los nodos están fijos- este procedimiento se repite hasta que se han reconectado todos los bordes. La probabilidad de reconexión $p$ está en función de los beneficios por especialización del comercio a distancia que se definen como:

$$
\text { beneficios por especialización }=\sqrt{1+\left(A P L_{0}-A P L_{t}\right)} \geq 1
$$

donde $A P L_{0}$ y $A P L_{t}$ son el estadístico $A P L$ al inicio y en el tiempo $t$ de la simulación, respectivamente. Al inicio de la simulación, cuando todas las relaciones son cercanas, $A P L_{0}$ y $A P L_{t}$ son iguales. Cuando comienzan a haber relaciones a distancia (o lazos débiles) $A P L_{t}$ disminuye. La probabilidad $p$ también está en función de costos de reconexión $C_{r}$ : para poder realizar comercio a distancia se requieren realizar proyectos de inversión en activo fijo y por ende en activo circulante o capital de trabajo; es decir, se deben desarrollar las comunicaciones y el transporte realizando la inversión adicional necesaria en carreteras, líneas ferroviarias, puertos marítimos, aeropuertos, medios de transporte terrestre, marítimo, aéreo, así como telegramas, correos, teléfonos, 
redes sociales; sin olvidar también la inversión adicional necesaria en efectivo, cuentas por cobrar, inventarios. Específicamente, la probabilidad $p$ está en función del margen del beneficio neto del comercio a distancia:

$$
p_{t+1}=2\left(\frac{\sqrt{1+\left(A P L_{0}-A P L_{t}\right)}-C_{r}}{\sqrt{1+\left(A P L_{0}-A P L_{t}\right)}}\right)
$$

donde $p(t+1)$ es la probabilidad de reconexión en el tiempo $t+1$ y el margen se multiplica por dos para que $p$ tome valores entre cero y uno. Cabe mencionar que cada vez que se promueve la reconexión de un lazo original (transacción relacional), se incurre en costos de reconexión $C_{r}$. Cierto es que $\frac{\partial p_{t+1}}{\partial A P L_{t}}>0$.

\subsection{Dinámicas de comportamiento}

Al inicio de la simulación, un porcentaje de los $N$ se comportan cooperativamente y los demás de forma oportunista; de tal forma que la probabilidad de cooperadores en el sembrado inicial es $N_{C o} \in(0,1)$.

Posteriormente cada individuo se involucra repetidamente en un juego del dilema del prisionero de múltiples jugadores. Se denota como $\Gamma_{(i, t)}$ al conjunto de compañeros de juego con los cuales el jugador $i$ interactúa en el periodo $t(=1,2,3, \cdots n)$. En cada interacción, además de suponerse una estructura de beneficios tipo dilema del prisionero, se supone que los jugadores observan sus resultados así como los resultados y estrategias de sus compañeros de juego. Basados en esta información, los jugadores aprenden a través del tiempo cuál es la estrategia a imitar. Los jugadores actualizan sus estrategias de comportamiento al final de cada intervalo de tiempo de forma probabilística y en función de beneficios relativos.

En cada periodo de tiempo el jugador $i$ puede escoger cooperar (C) o no cooperar (D) en las relaciones con sus vecinos, utilizando la misma estrategia con todo su vecindario. La matriz de beneficios asociada a los juegos bilaterales viene descrita de la siguiente manera:

\begin{tabular}{|c|c|c|}
\hline $\mathrm{i} / \mathrm{j}$ & $\mathrm{C}$ & $\mathrm{D}$ \\
\hline $\mathrm{C}$ & $(\mathrm{R}, \mathrm{R})$ & $(\mathrm{S}, \mathrm{T})$ \\
\hline $\mathrm{D}$ & $(\mathrm{T}, \mathrm{S})$ & $(\mathrm{P}, \mathrm{P})$ \\
\hline
\end{tabular}

donde $i$ y $j$ son los jugadores columna y renglón respectivamente (por ejemplo, si $i C$ y $j D, i$ recibe el resultado $S$ y $j$ recibe el resultado $T$ ). Escribiendo $\pi\left(a_{i}, a_{j}\right)$ como el resultado del jugador i por usar la estrategia $a_{i}$ cuando la estrategia del socio $j$ es $a_{j}$, se sigue que:

$$
\pi(C, C)=R, \pi(C, D)=S, \quad \pi(D, C)=T \quad y \quad \pi(D, D)=P
$$

Para que el juego implique un dilema social se requiere que $T>R>P>$ $S$ y también que $2 R>(T+S)$. La primera condición asegura que el no cooperar le dé al jugador un resultado más alto independientemente de cuál sea la estrategia del oponente. Por lo tanto, si el juego se fuera a jugar solamente una vez y los jugadores fueran racionales nadie cooperaría y el resultado obtenido sería sub-óptimo en términos sociales. La segunda condición implica 
que la cooperación mutua siempre es mejor, en el sentido de que ésta genera un resultado Pareto superior.

En el modelo:

- Se supone que el comercio que reditúa más es el comercio a distancia, por lo que la especialización incrementa el beneficio de los nodos independientemente si hay o no cooperación.

- También se supone que para poder realizar el comercio a distancia se incurren en costos de reconexión $C_{r}$ que disminuyen el beneficio del nodo que promueve la relación a distancia.

- Un individuo con capacidad económica para promover una transacción a distancia es aquel con una riqueza acumulada a través del tiempo igual o mayor a un cierto umbral $\Omega^{*}$. Un individuo con poder económico o persona "muy important" ( persona $_{V I P}$ ) es aquel con un número de relaciones a distancia igual o mayor a un cierto umbral $L_{d}^{*}$. Cuando el porcentaje de personas $s_{V I P}$ es igual o mayor a un cierto umbral $V I P^{*}$, se forma un grupo de poder económico o elite económica que permite a los individuos que la conforman estar en condiciones de comenzar a sancionar el comportamiento oportunista en sus relaciones.

- Cada periodo de tiempo los individuos suman los beneficios obtenidos en todas sus interacciones.

De los anteriores puntos, el beneficio neto total para el individuo $i$ en el periodo $t$ es:

$$
\Pi_{\mathrm{i}, \mathrm{t}}=\sum_{j \in \Gamma_{i, t}}\left[\pi\left(a_{i, t}, a_{j, t}\right) \sqrt{1+\left(A P L_{0}-A P L_{t}\right)}-C_{r}\right]\left(1-T_{p}\right)
$$

donde $\pi\left(a_{(i, t)}, a_{(j, t)}\right)$ es el resultado del individuo $i$ en el tiempo $t$ por usar la estrategia $a_{i}$ cuando la estrategia del socio $j$ es $a_{j} ; \sqrt{1+\left(A P L_{0}-A P L_{t}\right)}$ son los beneficios por especialización (ecuación 1); $C_{r}$ son los costos de reconexión; $T_{p}$ es la tasa de castigo al comportamiento oportunista. A la elite económica se van sumando los individuos que van cubriendo los requisitos económicos. En este sentido se dice que se crean instituciones de "abajo-arriba" en la economía, de tal forma que las transacciones de mercado no sean sujetos del oportunismo.

Para poder sancionar el comportamiento oportunista en las transacciones a distancia se requieren realizar inversiones destinadas a desarrollar instituciones de refuerzo e información. Estas instituciones tienen como objetivo reducir los costos de transacción para hacer posible la producción y el intercambio de mercancías y servicios en entornos de incertidumbre. Estos costos están asociados a los problemas de información asimétrica, especificidad de los activos (costos hundidos), racionalidad limitada y contratos incompletos, que hacen muy difícil eliminar de manera ex-ante los conflictos de intereses y el oportunismo que eventualmente pudieran surgir entre las partes involucradas en una transacción a distancia o de mercado.

El nivel de acumulación de riqueza intertemporal que incide en la capacidad para establecer relaciones a distancia del individuo $i$ en el periodo $t$ es:

$$
\Omega_{\mathrm{i}, \mathrm{t}}=\Omega_{\mathrm{i}, \mathrm{t}-1}=\Pi_{\mathrm{i}, \mathrm{t}}
$$


donde $\Omega_{(i, t-1)}$ es el nivel de acumulación de riqueza intertemporal para el individuo $i$ en el periodo $t-1$.

Los parámetros del modelo de investigación se muestran en el siguiente cuadro.

Tabla 1. Parámetros del modelo de investigación

\begin{tabular}{|c|c|c|}
\hline $\mathrm{N}$ & $(=40)$ & Tamaño de la población \\
\hline $\mathrm{k}$ & $(=4)$ & Grado de cada vértice en el enrejado de anillo regular \\
\hline$N_{C o}$ & $(=0.5)$ & Probabilidad de cooperadores en el sembrado inicial \\
\hline $\mathrm{T}$ & $(=1.6)$ & Resultado del egoísmo \\
\hline $\mathrm{R}$ & $(=1.0)$ & Resultado de la cooperación conjunta \\
\hline $\mathrm{P}$ & $(=0.1)$ & Resultado de la no cooperación conjunta \\
\hline $\mathrm{S}$ & $(=0.0)$ & Resultado del altruismo \\
\hline$p_{\pi}$ & $(=0.98)$ & Probabilidad de elegir la estrategia del vecino que \\
& & le fue mejor \\
\hline$\Omega^{*}$ & $(=10)$ & Umbral de riqueza \\
\hline$C_{r}$ & $(=0.99)$ & Umbral de número de lazos débiles \\
\hline$L_{d}^{*}$ & $(=6)$ & Tasa de castigo al comportamiento oportunista \\
\hline$V I P^{*}$ & $(=0.1)$ & Umbral de $\%$ de nodos con un número de lazos débiles $L_{d}^{*}$ \\
\hline$T_{p}$ & $(=0.3)$ &
\end{tabular}

Fuente:Elaboración propia.

\section{3 ¿Cómo opera el modelo?}

Al inicio de la simulación se observan cooperadores y no cooperadores en la población. Los individuos sólo realizan transacciones con gente cercana. Estas transacciones relacionales se llevan a cabo a través de una estructura tipo dilema del prisionero. Como los individuos observan tanto sus beneficios como las estrategias y beneficios de sus compañeros de juego, los primeros van aprendiendo qué estrategia les conviene imitar. En función de las estrategias seguidas cada individuo va acumulando riqueza.

Aquellos individuos que acumulan riqueza por encima de un cierto umbral comienzan a realizar transacciones a distancia con una probabilidad que está en función de lo que ellos consideran son los beneficios netos del comercio a distancia; teniendo en cuenta que el comercio a distancia brinda beneficios por especialización pero se incurren en costos para poder llevarlo a cabo.

Una vez que un individuo decide establecer una transacción a distancia, la conexión la hace de manera aleatoria con individuos que no están en su vecindad. Para establecer una relación a distancia el individuo debe sacrificar una relación cercana. Una vez que se establece una relación a distancia ésta se sostiene para siempre. Cada individuo puede promover tantas relaciones a distancia como relaciones cercanas tenga la persona.

Posteriormente, cuando algunos individuos alcanzan cierto poder económico a raíz de sus transacciones a distancia y este grupo representa un porcentaje importante de la población total, se forma un grupo de poder. 
En estas circunstancias ‘ los individuos que conforman la élite están en condiciones de comenzar a sancionar el comportamiento oportunista en sus relaciones. A este grupo de poder se van sumando los individuos que van cubriendo los requisitos económicos. Finalmente, como se supone una economía sin crecimiento poblacional, una vez que se han transformado todas las transacciones en anónimas, el sistema se detiene.

\section{Resultados}

En la Figura 1 se ilustra una economía tradicional al inicio de una simulación donde los individuos se ubican en los nodos de la red y los lazos de la red definen las conexiones que existen entre ellos. Se parte de una situación en la que cerca de la mitad de la población se comporta cooperativamente (nodos azules) y los actores realizan exclusivamente transacciones relacionales en un contexto de dilema del prisionero.

Figura 1. Economía tradicional al inicio de una simulación

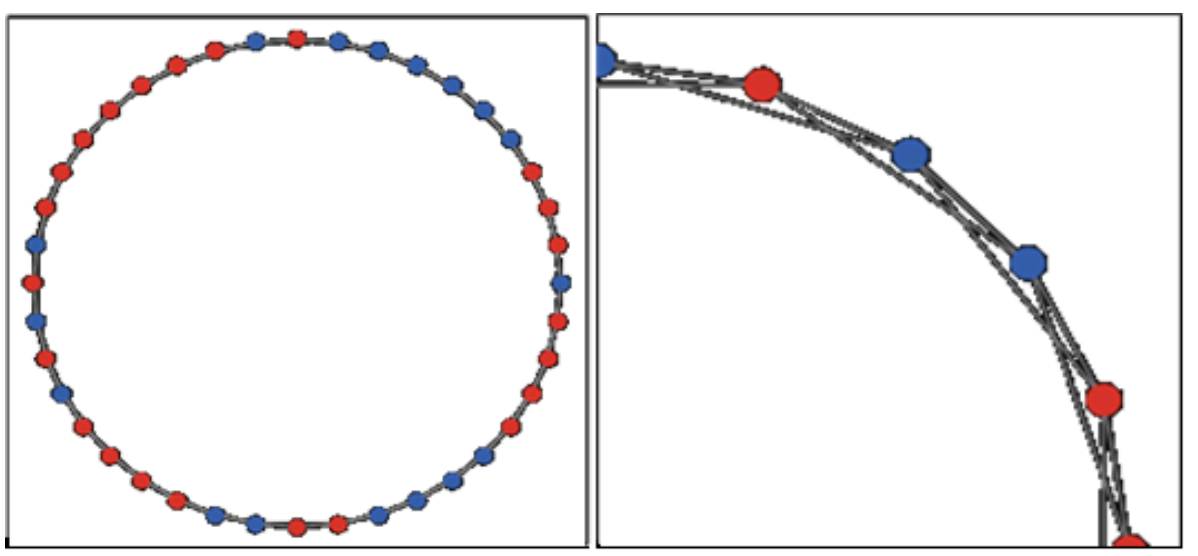

Fuente:Elaboración propia.

En la Figura 2 se ilustra la economía tradicional al final de una simulación, los individuos han aprendido a través del tiempo que estrategia les conviene imitar, la estrategia de comportamiento del compañero de juego que obtuvo un mejor resultado: cooperar. 
Figura 2. Economía tradicional al final de una simulación

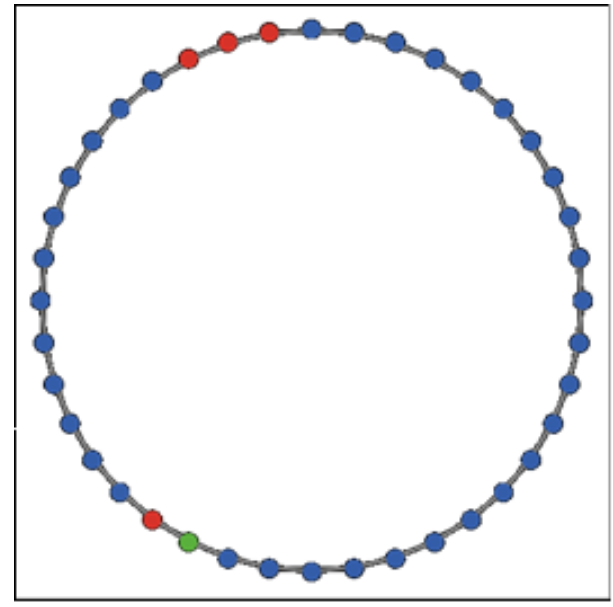

Fuente:Elaboración propia.

En la Figura 3 se ilustra una economía tradicional en el proceso de modernización. La red se ha transformando al haberse reconectado alrededor del 50 por ciento de los lazos originales (transacciones relacionales) de tal forma que el individuo que promueve la reconexión termina conectado ahora con una persona afuera de su vecindario (transacciones a distancia). La consecuente disminución en la cohesión social ha terminado por promover el comportamiento oportunista (nodos rojos) en la economía.

Figura 3. Economía tradicional en el proceso de modernización

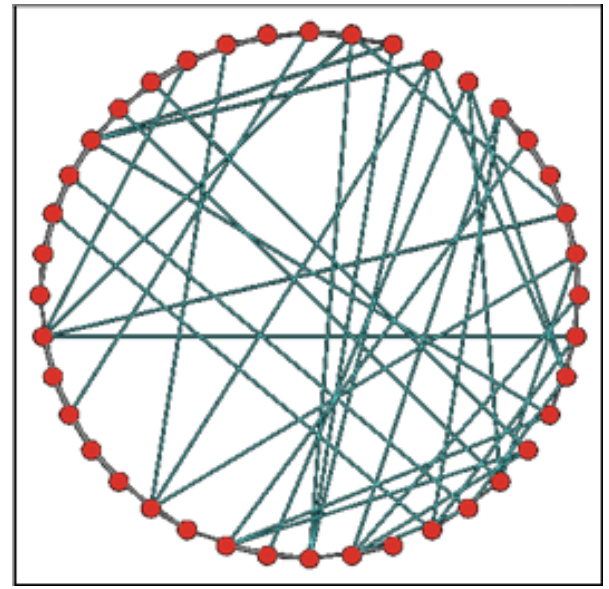

Fuente:Elaboración propia.

En la Figura 4 se ilustra una economía moderna en la que todas las transacciones son a distancia pero en la que también las elites económicas castigan el oportunismo en sus relaciones, promoviendo así la cooperación en la economía. 
Figura 4. Economía moderna

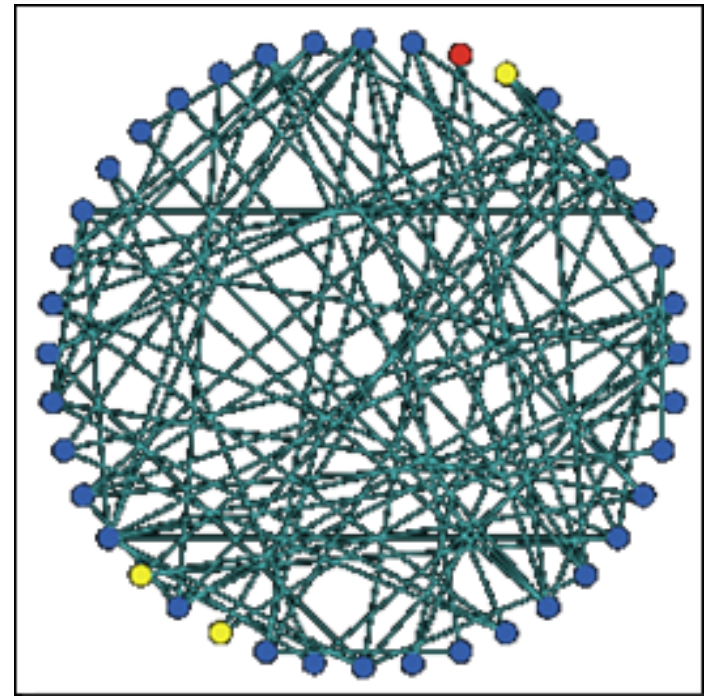

Fuente:Elaboración propia.

La riqueza individual (ver la Gráfica 1 donde INCF es la riqueza individual) y la cooperación (Gráfica 2), una vez iniciada la simulación, disminuyen hasta la mitad de la simulación, aunque ambas variables crecen luego hasta el final de la simulación. A su vez, tiempo después de iniciada la simulación el número de personas con poder económico (Gráfica 3) crece hasta el final de la simulación.

Gráfico 1. Medias a través de la simulación del INCF

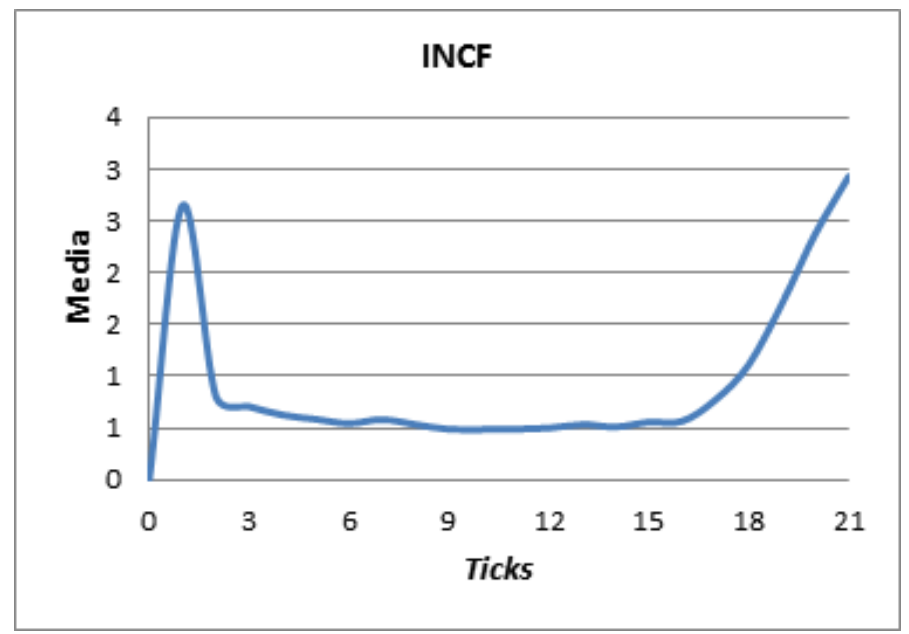

*Medias de 40 simulaciones (ver la Tabla 1 en el Apéndice). 
Gráfico 2. Medias a través de la simulación del porcentaje de cooperadores

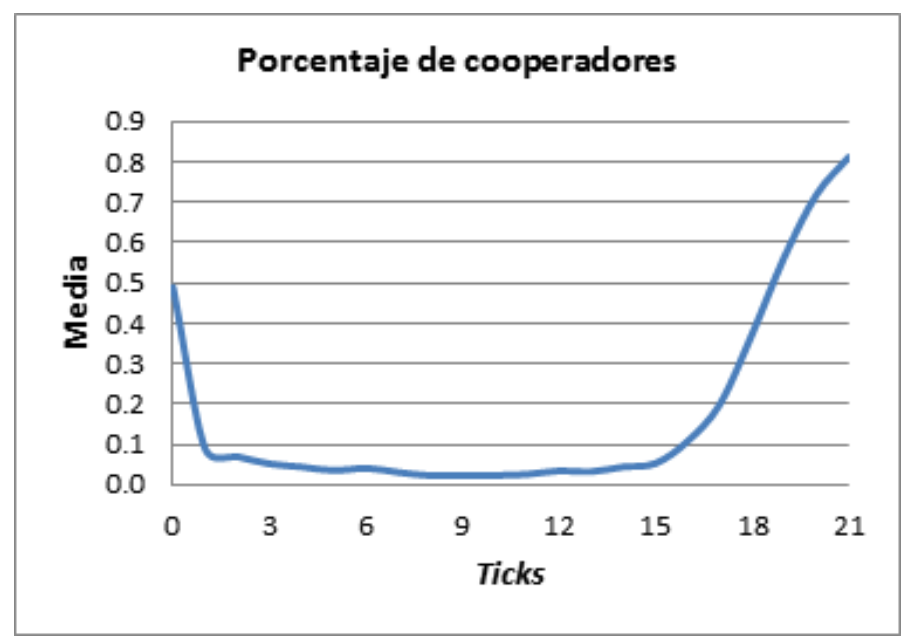

*Medias de 40 simulaciones (ver la Tabla 1 en el Apéndice).

Gráfico 3. Medias a través de la simulación del número de personas $V I P$

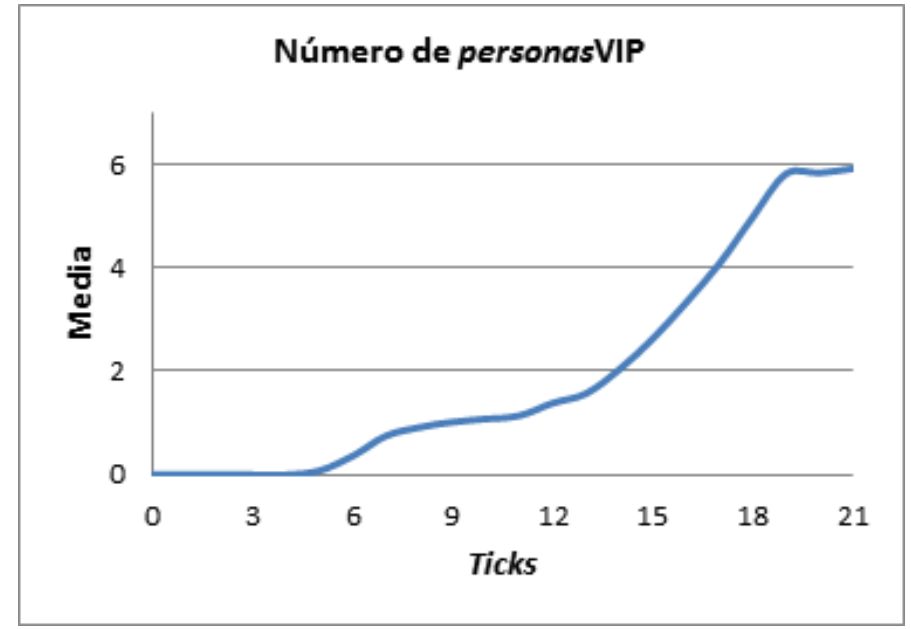

*Medias de 40 simulaciones (ver la Tabla 1 en el Apéndice).

En otras palabras, cuando las transacciones son relacionales, la riqueza individual crece con todo y la cooperación. Luego, cuando comienzan a haber transacciones a distancia la riqueza individual decrece con todo y la cooperación. Eventualmente, la riqueza individual repunta con todo y la cooperación, cuando también repunta el número de personas con poder económico. ¿Por qué pasa todo ésto? Cuando las transacciones son relacionales, el elevado nivel de clustering facilita el que se siga una estrategia cooperativa y esto se refleja 
en un incremento en la riqueza individual, al disminuir el oportunismo en las transacciones. Luego las transacciones a distancia rompen el clustering y por consiguiente la cooperación, y esto se refleja en una disminución en la riqueza individual, a pesar de los beneficios netos de la especialización que también conlleva el comercio a distancia. Sin embargo, la riqueza individual que se va acumulando con la especialización permite la formación de elites económicas que comienzan a sancionar el oportunismo en sus relaciones, lo que lleva a la acumulación de aún más riqueza y al crecimiento de estas elites. Finalmente el orden regresa vía la sanción y despega la economía.

Tiempo después de iniciada la simulación la riqueza individual tanto para las personas con poder económico, como para las personas "comunes" (ver la Gráfica 4 donde $I N C F_{V I P}$ es la riqueza individual para las personas con poder económico y $I N C F_{V C P}$ es la riqueza individual para las personas "comunes"), crece hasta el final de la simulación, siendo la riqueza de los primeros cada vez mayor a la de los segundos. Por qué? Cuando las elites comienzan a sancionar el oportunismo en sus relaciones, comienza a crecer la brecha entre la riqueza individual de los miembros de estas elites y la riqueza individual de las personas que no forman parte de estos grupos de poder, quedando la riqueza de los primeros muy por encima de la de los segundos.

Gráfico 4. Medias a través de la simulación: $I N C F_{V I P}$ y $I N C F_{V C P}$

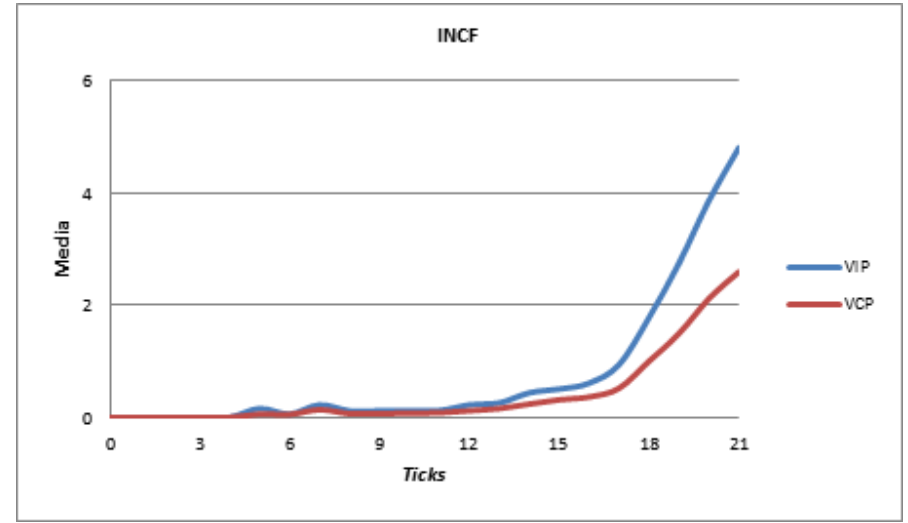

*Medias de 40 simulaciones (ver la Tabla 2 en el Apéndice).

A través de la simulación, las relaciones entre medias y varianzas de las variables de estudio muestran que la dispersión de los datos con respecto a su media, no aumenta conforme aumenta la media en la simulación y viceversa (Gráficas 5 y 6 ). 
Gráfica 5. Medias y varianzas a través de la simulación: INCF.

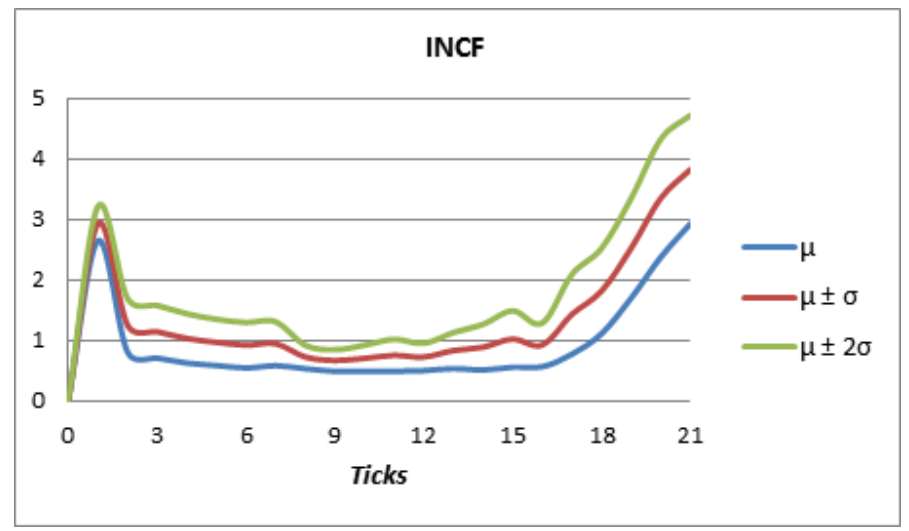

*Estadísticos de 40 simulaciones (ver la Tabla 2 en el Apéndice).

Gráfica 5. Medias y varianzas a través de la simulación: Número de personas ${ }_{V I P}$

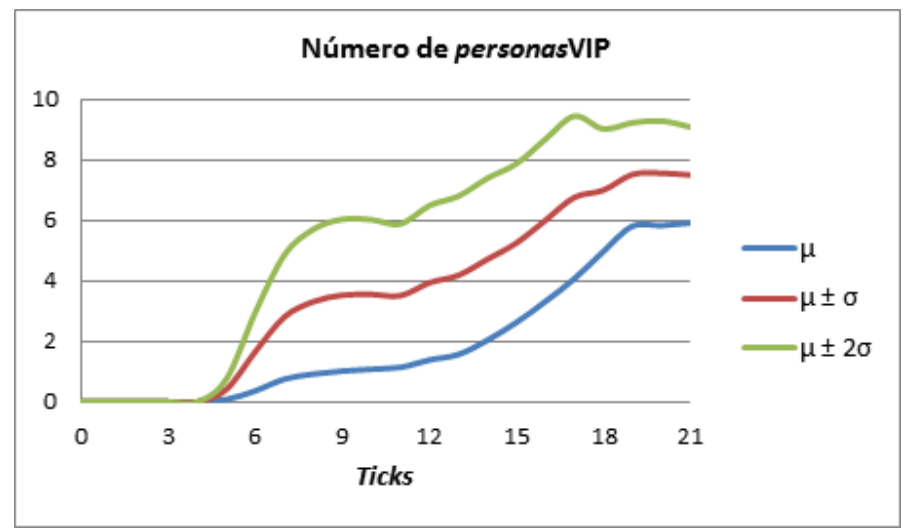

*Estadísticos de 40 simulaciones (ver la Tabla 2 en el Apéndice). 
142 Nueva Época REMEF (The Mexican Journal of Economics and Finance)

Gráfico 5. Medias y varianzas a través de la simulación: Porcentaje de cooperadores

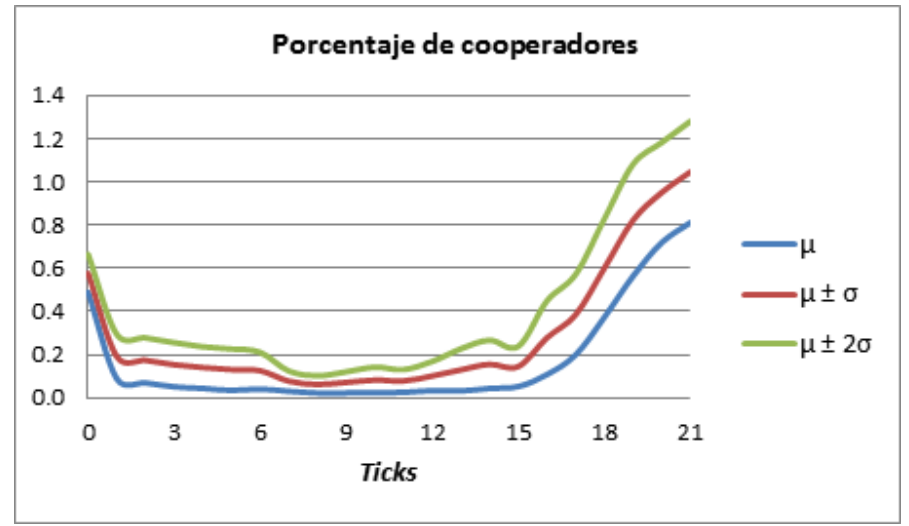

*Estadísticos de 40 simulaciones (ver la Tabla 2 en el Apéndice).

Gráfico 6. Medias y varianzas a través de la simulación: $I N C F_{V I P}$

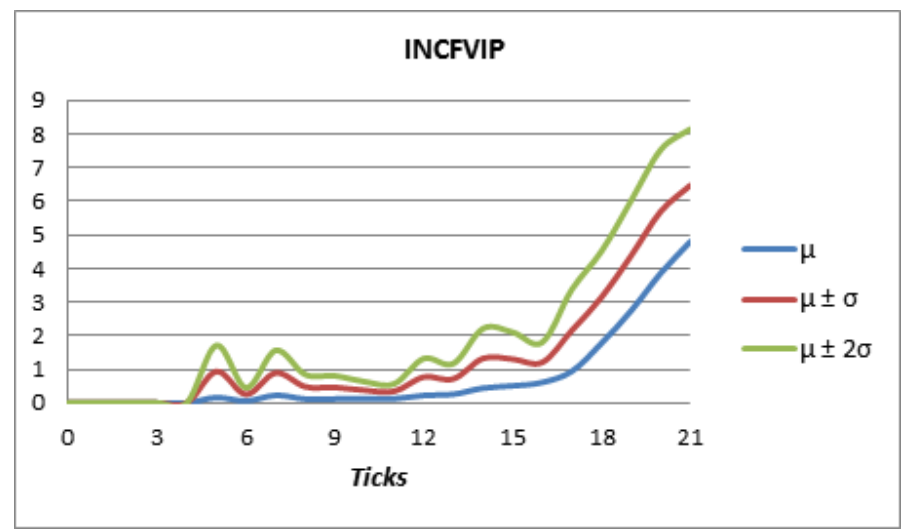

*Estadísticos de 40 simulaciones (ver la Tabla 2 en el Apéndice). 
Gráfico 6. Medias y varianzas a través de la simulación: $I N C F_{V C P}$

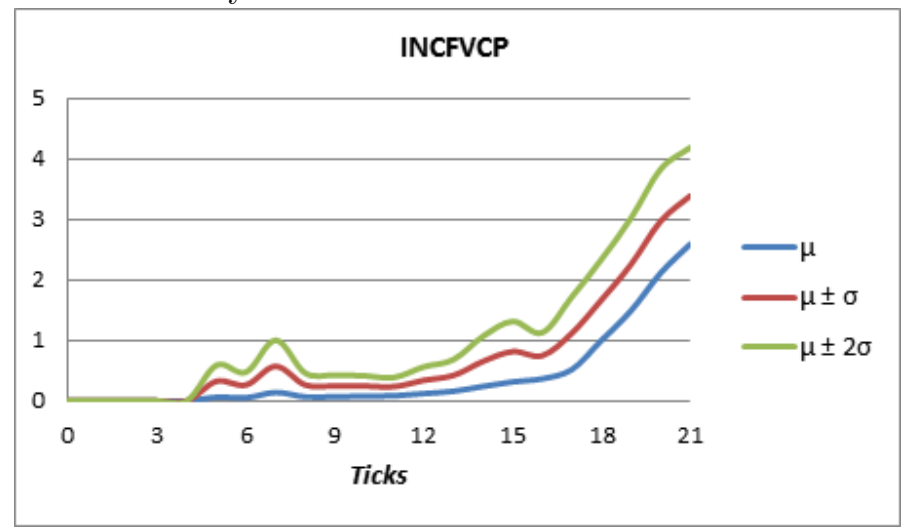

*Estadísticos de 40 simulaciones (ver la Tabla 2 en el Apéndice).

Como se ilustra en la Gráfica 7, la cooperación y el umbral de acumulación de riqueza $\Omega^{*}$ (que se requiere alcanzar para poder establecer una relación a distancia) se relacionan positivamente. ¿Por qué? Cuando la economía es relacional, con el paso del tiempo, la mayoría de los individuos aprenden a comportarse cooperativamente, de tal forma que, entre más tarden los individuos en comenzar a realizar transacciones a distancia, más tenderán a propagar, de entrada, la cooperación en sus nuevas relaciones.

Gráfica 7. Sensibilidad del porcentaje de cooperación final a diferentes umbrales de acumulación

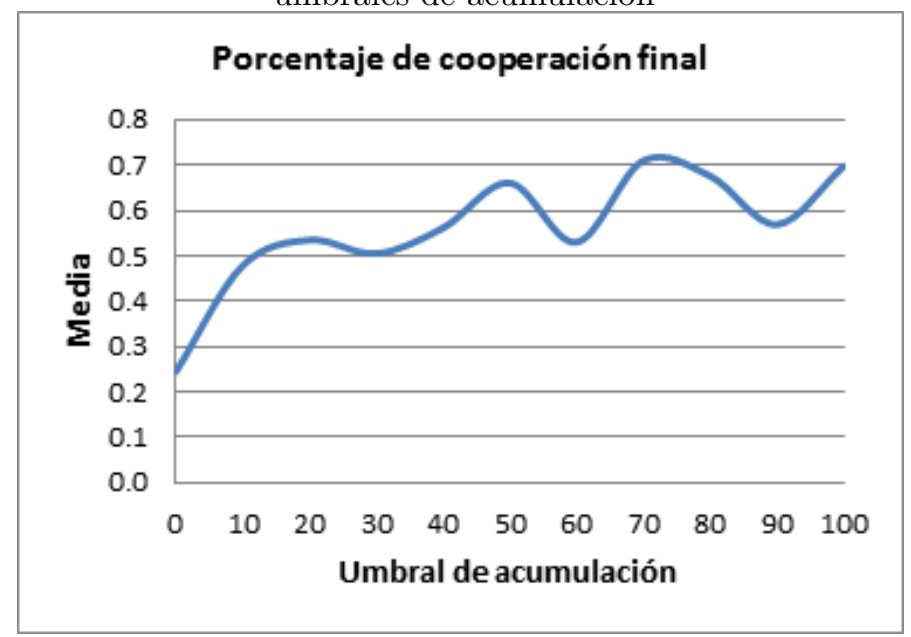

*Medias de 40 simulaciones (ver la Tabla 3 en el Apéndice).

Como se muestra en la Gráfica 8 , la cooperación y la tasa de castigo $T_{p}$ (a la que se gravan los resultados de los oportunistas) se relacionan positivamente: entre más fuertemente se castigue al oportunismo en las relaciones, más contundentemente se promoverá la cooperación en la economía. 
144 Nueva Época REMEF (The Mexican Journal of Economics and Finance)

Gráfica 8. Sensibilidad del porcentaje de cooperación final a diferentes tasas de castigo

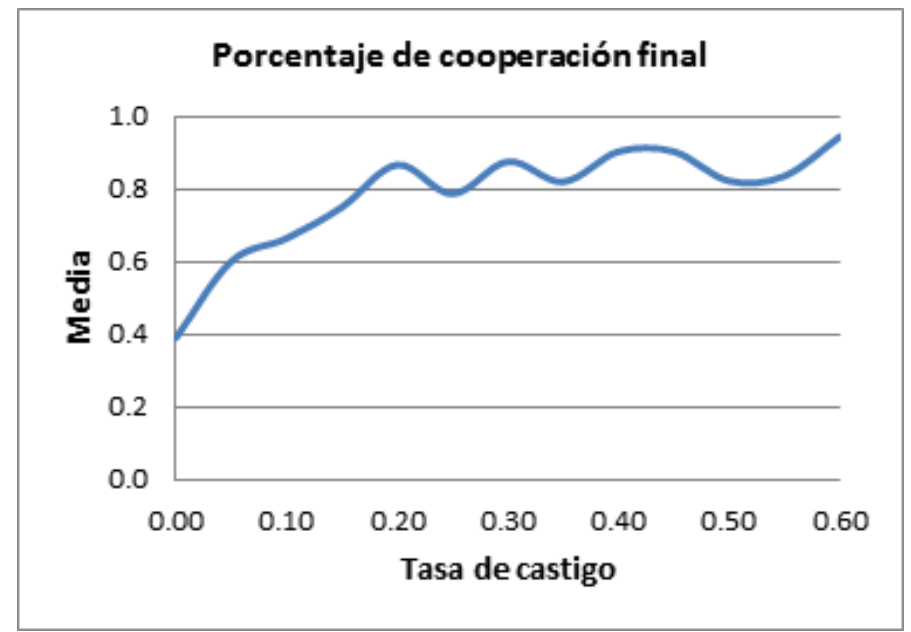

*Medias de 40 simulaciones (ver la Tabla 3 en el Apéndice).

Como se observa en la Gráfica 9, la cooperación y el umbral del número de relaciones a distancia $L_{d}^{*}$ (que se requiere alcanzar para ser una persona con poder económico) se relacionan negativamente: entre menos relaciones a distancia se requieran tener para ser una persona con poder económico, más pronto se formarán elites económicas que comenzarán a sancionar el oportunismo y a propagar la cooperación en sus relaciones y en las relaciones de los demás, antes de que lo que se propague en la economía sea el oportunismo.

Gráfico 9. Sensibilidad del porcentaje de cooperación final a diferentes umbrales de relaciones

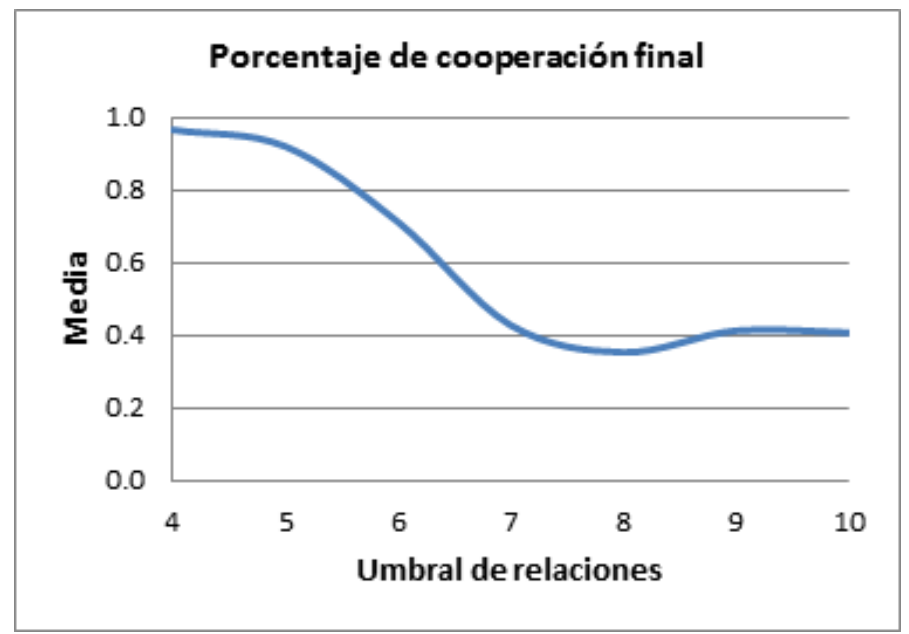

*Medias de 40 simulaciones (ver la Tabla 3 en el Apéndice). 
Como se ve en la Gráfica 10, la cooperación y el umbral del porcentaje de personas con poder económico VIP* (que se requiere alcanzar para que se comience a sancionar el oportunismo en las relaciones de estas personas) se relacionan negativamente: entre menos personas con poder económico se requieran para comenzar a sancionar el oportunismo y propagar la cooperación en las relaciones, más se ganará la batalla al oportunismo en la economía.

Gráfico 10. Sensibilidad del porcentaje de cooperación final a diferentes umbrales de personas $V I P$

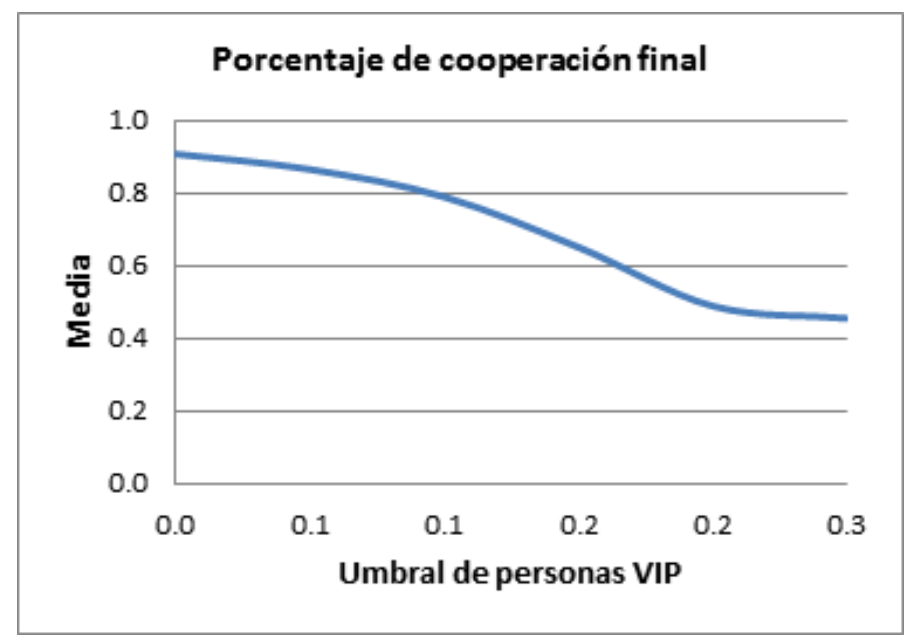

*Medias de 40 simulaciones (ver la Tabla 3 en el Apéndice).

\section{Conclusiones}

El presente trabajo de investigación tiene como objetivo explicar el desempeño de una sociedad en su proceso de modernización económica. Una economía tradicional se caracteriza por transacciones relacionales y grupos con un alto grado de cohesión social que dan lugar a creencias e intereses compartidos. En cambio en una economía moderna los mercados son anónimos y existe un grado de cohesión social insignificante, es decir la economía se caracteriza por interacciones a distancia.

La hipótesis de investigación que se propone en el artículo es la siguiente. Cuando la organización en una sociedad es altamente cohesionada y el comercio a distancia es casi inexistente se preserva la cooperación de la comunidad pero se retarda el desarrollo del comercio. Así mismo, cuando estas comunidades comienzan a incrementar las interacciones económicas con forasteros se desarrolla el comercio pero también se produce un fallo en la cohesión de la comunidad y un fracaso en la cooperación y acciones colectivas emprendidas por los miembros de la comunidad. Para evitar que la cooperación colapse deben surgir instituciones de terceros en la sociedad que refuercen el comportamiento cooperativo, lo que permite disminuir el oportunismo en los intercambios comerciales y hacer posible el despegue de la economía.

En la investigación se modela el proceso de construcción de una economía de mercado (transacciones a distancia). Partiendo de una situación en donde 
cerca de la mitad de la población se comporta cooperativamente y en donde la economía es tradicional, los actores realizan exclusivamente transacciones relacionales en un contexto de dilema del prisionero. Con el paso del tiempo los jugadores van aprendiendo cuál estrategia de comportamiento les conviene más al observar la estrategia de comportamiento del compañero de juego que obtuvo un resultado mayor. Se supone que el comercio a distancia reditúa beneficios adicionales por la especialización, a pesar de que es necesario invertir en bienes de capital para que el mismo se lleve a cabo. De esta manera, con una probabilidad que está en función del beneficio neto del comercio a distancia, aquellos individuos que han acumulado un cierto nivel de riqueza comienzan a establecer relaciones a distancia sacrificando relaciones cercanas. En el modelo se hace referencia a la creación de instituciones o sanción entre terceros cuando el porcentaje de individuos con un cierto poder económico alcanza cierto umbral, lo que permite sancionar el oportunismo en las relaciones de estas personas; de esta manera las instituciones en la economía surgen de "abajo-arriba".

El modelo que aquí se elabora propone un juego evolutivo espacial en el que los individuos se ubican en los nodos de una red endógena y los lazos de la red definen las conexiones que existen entre ellos. Paulatinamente, la red se va transformando cuando se van reconectando los lazos originales (transacciones relacionales) de tal forma que el individuo que promueve la reconexión termina conectado ahora con una persona afuera de su vecindario (transacciones anónimas o a distancia). De esta manera, los individuos se involucran en cada periodo en un juego del dilema del prisionero con múltiples jugadores. Los jugadores aprenden a través del tiempo que estrategia les conviene, por lo que imitan la estrategia de comportamiento del compañero de juego que obtuvo un mejor resultado.

Los resultados del trabajo se pueden resumir como sigue. Cuando las transacciones son relacionales, el elevado nivel de clustering facilita el que se siga una estrategia cooperativa y esto se refleja en un incremento en la riqueza individual, al disminuir el oportunismo en las transacciones. Luego las transacciones a distancia rompen el clustering y por consiguiente la cooperación, y esto se refleja en una disminución en la riqueza individual, a pesar de los beneficios netos de la especialización que también conlleva el comercio a distancia. Sin embargo, la riqueza individual que se va acumulando con la especialización permite la formación de elites económicas que comienzan a sancionar el oportunismo en sus relaciones, lo que lleva a la acumulación de aún más riqueza y al crecimiento de estas elites. Finalmente el orden regresa vía la sanción y despega la economía. En particular, cuando las elites económicas comienzan a sancionar el oportunismo en sus relaciones, comienza a crecer la brecha entre la riqueza de los miembros de estas elites y la de los individuos que no forman parte de estos grupos de poder, quedando la riqueza de los primeros muy por encima de la de los segundos.

a) La razón por la cual países severamente subdesarrollados están a menudo fragmentados en divisiones profundas sobre la base de criterios etnolingüísticos, se puede deber a que este es un arreglo necesario para asegurar un intercambio económico honesto mínimo hacia adentro del grupo.

b) Si el país se moderniza demasiado rápido y las interacciones entre individuos, a través de grupos, se incrementan más rápido que el surgimiento de 
intermediación de terceros confiable, el resultado puede ser la anarquía económica.

c) Después de que se cruza un cierto umbral en el proceso de modernización, el surgimiento de intermediación de terceros confiable viene a ser esencial para desarrollar los mercados y el intercambio, más allá de dicho umbral.

\section{Bibliografía}

Acemoglu, D. y J. A Robinson (2005). Economic Origins of Dictatorship and Democracy. Cambridge University Press.

Anand, S. y A. Sen (1994). Sustainable Human Development: Concepts and Priorities. New York: United Nations Development Programme. Discussion Papers Series.

Andreoni, J. (1988). Privately provided Public Goods in a Large Economy: the Limits of Altruism. Journal of Public Economics 35, pp. 57-73.

Andreoni, J. (1990). Impure Altruism and Donations to Public Goods: A Theory of Warmglow Giving. Economic Journal 100, pp. 467-77.

Becker, G. (1985). Public Policies, Pressure Groups, and Deadweight Costs. Journal of Public Economics, 28, pp. 329-47.

Bernhard, M. (1993). Civil Society and Democratic Transitions in East Central Europe. Political Science Quarterly, 108, pp. 307-26.

Bianco, W.T. y R. H Bates (1990). Cooperation by design: Leadership, Structure, and Collective Dilemmas. American Political Science Review, 84 pp. 133-47.

Blomkvist, H. (2003). Social Capital, Political participation and the Quality of Democracy in India. Artículo preparado para la reunión anual de la Asociación de Ciencia Política Americana, en Filadelfia.

Boix, C. y S. C. Stokes (2003). Endogenous Democratization. World Politics 55, pp. 517-49.

Bollen, K.A. y R. W. Jackman (1985). Political Democracy and the size Distribution of Income. American Sociological Review, 50, pp. 438-57.

Bollen, K.A. y P. M. Paxton (2000). Subjective Measures of Liberal Democracy. Comparative Political Studies, 33, pp. 58-86.

Brint, S. (1984). 'New class' and Cumulative Trend Explanations of the Liberal Political Attitudes of Professionals. American Journal of Sociology, 90, pp. 30-71.

Bunce, V. (2000). Comparative Democratization: Big and Bounded Generalizations. Comparative Political Studies, 33, pp. 703-34.

Burkhart, R. E. y Lewis-Beck, M. S. (1994). Comparative Democracy: The Economic Development Thesis. American Political Science Review, 88, pp. 903-10.

Caporaso, J.A. y D. P. Levine (1992). Theories of Political Economy. Cambridge University Press.

Castañeda, G. (2009). Sociomática: el estudio de los sistemas adaptable complejos en el entorno socioeconómico. Trimestre Económico, 301, pp. 5-64.

Chamberlin, J. (1974). Provision of Collective Goods as a Function of Group Size. American Political Science Review, 68, pp. 707-16.

Coleman, J.S. (1988). Social Capital and the Creation of Human Capital. American Journal of Sociology, 94 pp. S95-S120.

Cornes, R. y T. Sandler (1984). Easy Riders, Joint Production, and Collective Action. Economic Journal, 94, pp. 580-98.

Doorenspleet, R. (2004). The Structural Context of Recent Transitions to Democracy. European Journal of Political Research, 43, pp. 309-36.

Eckstein, H. S. (1988). A Culturalist Theory of Political Change. AAmerican Political Science Review, 82, pp. 789-804.

Elkins, Z. (2000). Gradations of Democracy? Empirical Tests of Alternative Conceptualizations. American Journal of Political Science, 44, pp. 287-94.

Estes, R.J. (2000). Social Development Trends in the Middle East, 1970-1997: The Search for Modernity. Social Indicators Research, 50, pp. 51-80. 
Flanagan, S. e R. Inglehart (1987). Value Change in Industrial Society. American Political Science Review, 81 pp.1289-1319.

Förster, J., E. T. Higgins e L. C. Idson (1998). Approach and Avoidance Strength during Goal Attainment: Regulatory Focus and the 'Goal Looms Larger' effect. Journal of Personality and Social Psychology, 75, pp. 1115-31.

Frohlich, N. y J. A. Oppenheimer (1970). I get by with a little help from my friends. World Politics 23, pp. 104-20

Fukuyama, F. (1989). The End of History. National Interest, 16, pp. 4-18.

Geddes, B. (1999). What do We Know about Democratization after Twenty Years? Annual Review of Political Science, 2, pp. 115-44.

Gibson, J.L. (2001). Social Networks, Civil Society, and the Prospects for Consolidating Russia's Democratic Transition. American Journal of Political Science, 45, pp. 51-69.

Granovetter, M. (1978). Threshold Models of Collective Behavior. American Journal of Sociology, 83, pp. 1420-43.

Guttman, J. M. (1978). Understanding Collective Action: Matching Behavior. American Economic Review, 68, pp. 251-55.

Haggard, S. y R. R. Kaufman (1995). The Political Economy of Democratic Transitions. Princeton University Press.

Heckathorn, D. D. (1988). Collective Sanctions and the Creation of Prisoner's Dilemma Norms. American Journal of Sociology, 94, pp. 535-62.

Heckathorn, D. D. (1990). Collective Sanctions and Compliance Norms: A Formal Theory of Group Mediated Social Control. American Sociological Review, 55, pp. 366-84.

Helliwell, J. F. (1993). Empirical Linkages between Democracy and Economic Growth. British Journal of Political Science, 24, pp. 225-48.

Higley, J. y M. G. Burton (1989). The Elite Variable in Democratic Transitions and Breakdowns. American Sociological Review, 54, pp. 17-32.

Hofferbert, R.L. y H-D Klingemann (1999). Remembering the Bad old Days: Human Rights, Economic Conditions, and Democratic Performance in Transitional Regimes. European Journal of Political Research, 36, pp. 155-74.

Huntington, S. P. (1984). Will more Countries become Democratic? Political Science Quarterly, 99, pp. 193-218.

Isaac, R.M. y J. M. Walker (1988). Group Size Effects in Public Goods Provision: The Voluntary Contribution Mechanism. Quarterly Journal of Economics, 103, pp. 179-99.

Iversen, T. (1994). Political Leadership and Representation in Western European Democracies: A Test of Three Models of Voting. American Journal of Political Science, 38, pp. 46-74.

Jenkins, J. C. (1983). Resource Mobilization Theory and the Study of Social Movements. Annual Review of Sociology, 9, pp. 527-33.

Johnson, N. R. y W. E. Feinberg (1977). A Computer Simulation of the Emergence of Consensus in Crowds. American Sociological Review, 42 pp. 505-21.

Kaizuku, K. (1965). Public Goods and Decentralization of Production. Review of Economics and Statistics, 47, pp. 118-20.

Kali, R. (2003). Social Embeddedness and Economic Governance: A Small World Approach. University of Arkansas.

Karklins, R. y R. Petersen (1993). Decision Calculus of Protestors and Regimes: Eastern Europe 1989. Journal of Politics 55, pp. 588-614.

Klandermans, B. (1984). Mobilization and Participation. American Sociological Review, 49, pp 583-600.

Knack, S. y Keefer, P. (1997). Does Social Capital have an Economic Payoff? A Cross-Country Investigation. Quarterly Journal of Economics, 112, pp. 1251-88.

Kopstein, J. S. y D. A. Reilly (2000). Geographic Diffusion and the Tansformation of the Post Communist World. World Politics, 53, pp. 1-37.

Kuran, T. (1991). Now Out of Never: The Element of Surprise in the East European Revolution of 1989. World Politics, 44, pp. 7-48. 
Kurzman, C. (1998). Waves of Democratization. Studies in Comparative International Development, 33, pp. 42-64.

Laumann, E.O., P. V Marsden y J. Galaskiewicz, J. (1977). Community -Elite Influence Structures: Extension of a Network Approach. American Journal of Sociology, 83, pp. 594-631.

Lipset, S.M. (1959). Some Social Requisites of Democracy: Economic Development and Political Legitimacy. American Political Science Review, 53: pp. 69-105.

Lipset, S. M. (1994). The Social Requisites of Democracy Revisited: 1993 Presidential Address. American Sociological Review, 59, pp. 1-22.

Macy, M. (1990). Learning Theory and the Logic of Critical Mass. American Sociological Review 55., pp. 809-26.

Macy, M. (1991a). Chains of Cooperation: Threshold Effects in Collective Action. American Sociological Review, 56, pp. 730-47.

Macy, M. (1991b). Learning to Cooperate: Stochastic and Tacit Collusion in Social Exchange. American Journal of Sociology, 92, pp. 808-43.

Marsden, P. V. (1981). Introducing Influence Processes into a System of Collective Decisions. American Journal of Sociology, 86, pp. 1203-35.

Marwell, G., P. E. Oliver and R Prahl (1988). Social Networks and Collective Action: A Theory of the Critical Mass. III. American Journal of Sociology, 94, pp. 502-34.

McAdam, D. (1983). Tactical Innovation and the Pace of Insurgency. American Sociological Review, 48, pp. 735-54.

McCarthy, J. D. y M. Zald (1977). Resource Mobilization and Social KMovements. American Journal of Sociology, 82, pp. 1212-42.

McPhail, C. y R. T. Wohlstein (1986). Collective Locomotion. American Sociological Review, 51, pp. 447-63.

Modelski, G. y G. Perry III (1991). Democratization in Long Perspective. Technological Forecasting and Social Change, 39, pp. 23-34.

Moore, B. (1993). Social Origins of Dictatorship and Democracy. Beacon Press.

Mouzelis, N. (1999). Modernity: A Non-European Conceptualization. British Journal of Sociology, 50, pp. 141-59.

Mueller, D.C. (1989). Public Choice II. Cambridge University Press.

Mueller, D.C. (1997). Perspectives on Public Choice. Cambridge University Press.

Muller, E.N. y M. A. Seligson (1994). Civic Culture and Democracy: The Question of Causal Relationships. American Political Science Review, 88, pp. 635-52.

O'Donnell, G. (1993). On the State, Democratization and some Conceptual Problems. World Development, 21, pp. 1355- 69.

O'Donnell, G. y P. C. Schmitter (1986). Transitions from Authoritarian Rule. Tentative Conclusions about Uncertain Democracies. The Johns Hopkins University Press.

Olson, M. (1965). The Logic of Collective Action. Harvard University Press.

Olson, M. (1982). The Rise and Decline of Nations. Yale University Press.

Olzak, S. (1989). Analysis of Events in the Study of Collective Action. Annual Review of Sociology, 15, pp. 119-41.

Paxton, P. (2002). Social Capital and Democracy: An Interdependent Relationship. American Sociological Review, 67, pp. 254-77.

Pitcher, B.L., R. L. Hamblin; y J. L. Miller (1978). The Diffusion of Collective Violence. American Sociological Review, 43, pp. 23-35.

Pye, L.W. (1990). Political Science and the Crisis of Authoritarianism. American Political Science Review, 84, pp. 3-19.

Runge, C.F. (1984). Institutions and the Free Rider: The Assurance Problem in Collective Action. Journal of Politics, 46, pp. 154-81.

Samuelson, P. A. (1954). The Pure Theory of Public Expenditure. Review of Economics and Statistics, 36, pp. 387-89.

Sides, J. (1999). It Takes Two: The Reciprocal Relationship between Social Capital and Democracy. Artículo preparado para la reunión anual de la Asociación de Ciencia Política Americana, en Atlanta. 
150 Nueva Época REMEF (The Mexican Journal of Economics and Finance)

Shin, D.C. (1994). On the Third Wave of Democratization: A Synthesis and Evaluation of Recent Theory and Research. World Politics 47, pp. 135-70.

Smith, A. (1976). The Wealth of Nations. University of Chicago Press.

Smith, J. (1976). Communities, Associations and the Supply of Collective Goods. American Journal of Sociology, 82, pp. 291-308.

Snyder, D. y C. Tilly (1972). Hardship and Collective Violence in France, 1830-1960. American Sociological Review, 37, pp. 520-32.

Sullivan, J.L. y J. E. Transue (1999). The Psychological underpinnings of Democracy: A Selective Review of Research on Political Tolerance, Interpersonal Trust, and Social Capital. Annual Review of Psychology, 50, pp. 625-50.

Tilly, C. (2004). Social Movements, 1768-2004. Paradigm Publishers, LLC.

Tabla 1 Medias y varianzas a través de la simulación: INCF, número de personas $_{V I P}$ y porcentaje de cooperadores

\begin{tabular}{|c|c|c|c|c|c|c|}
\hline \multirow[b]{2}{*}{ Tick } & \multicolumn{2}{|c|}{ INCF } & \multicolumn{2}{|c|}{$\begin{array}{c}\text { Número de } \\
\text { personas }_{V I P}\end{array}$} & \multicolumn{2}{|c|}{ Porcentaje de cooperadores } \\
\hline & Media & VAR & Media & VAR & Media & VAR \\
\hline 0 & S - & S - & 0 & 0 & 0.490 & 0.008 \\
\hline 1 & 2.65 & 0.08 & 0 & 0 & 0.088 & 0.010 \\
\hline 2 & 0.80 & 0.20 & 0 & 0 & 0.069 & 0.011 \\
\hline 3 & 0.71 & 0.19 & 0 & 0 & 0.052 & 0.010 \\
\hline 4 & 0.63 & 0.17 & 0 & 0 & 0.044 & 0.009 \\
\hline 5 & 0.59 & 0.15 & 0.08 & 0.12 & 0.035 & 0.009 \\
\hline 6 & 0.55 & 0.14 & 0.37 & 1.70 & 0.041 & 0.007 \\
\hline 7 & 0.58 & 0.13 & 0.76 & 4.23 & 0.031 & 0.002 \\
\hline 8 & 0.53 & 0.04 & 0.92 & 5.75 & 0.022 & 0.002 \\
\hline 9 & 0.49 & 0.03 & 1.02 & 6.31 & 0.022 & 0.002 \\
\hline 10 & 0.49 & 0.05 & 1.08 & 6.12 & 0.023 & 0.004 \\
\hline 11 & 0.49 & 0.07 & 1.14 & 5.63 & 0.026 & 0.003 \\
\hline 12 & 0.50 & 0.05 & 1.39 & 6.52 & 0.034 & 0.005 \\
\hline 13 & 0.53 & 0.09 & 1.57 & 6.86 & 0.032 & 0.01 \\
\hline 14 & 0.51 & 0.14 & 2.04 & 7.18 & 0.044 & 0.012 \\
\hline 15 & 0.56 & 0.22 & 2.63 & 6.89 & 0.053 & 0.009 \\
\hline 16 & 0.57 & 0.13 & 3.33 & 7.20 & 0.108 & 0.029 \\
\hline 17 & 0.77 & 0.44 & 4.08 & 7.22 & 0.199 & 0.035 \\
\hline 18 & 1.11 & 0.50 & 4.98 & 4.10 & 0.373 & 0.052 \\
\hline 19 & 1.70 & 0.68 & 5.82 & 2.93 & 0.564 & 0.067 \\
\hline 20 & 2.38 & 0.95 & 5.84 & 2.99 & 0.719 & 0.054 \\
\hline 21 & 2.93 & 0.81 & 5.92 & 2.52 & 0.812 & 0.055 \\
\hline
\end{tabular}

*Estadísticos de 40 simulaciones. 
Tabla 2. Medias y varianzas a través de la simulación: $I N C F_{V I P}$ y $I N C F_{V C P}$

\begin{tabular}{|c|c|c|c|c|}
\hline Tick & $\begin{array}{c}\text { Media } \\
\text { INCFvIP }\end{array}$ & $\begin{array}{c}\text { VAR } \\
\text { INCFvIP }\end{array}$ & $\begin{array}{c}\text { Media } \\
\text { INCFvcP }\end{array}$ & $\begin{array}{c}\text { VAR } \\
\text { INCFvcP }\end{array}$ \\
\hline 0 & $\$-$ & $\$-$ & $\$-$ & $\$$ \\
\hline 1 & - & - & - & - \\
\hline 2 & - & - & - & - \\
\hline 3 & - & - & - & - \\
\hline 4 & - & - & - & - \\
\hline 5 & 0.16 & 0.60 & 0.06 & 0.07 \\
\hline 6 & 0.06 & 0.04 & 0.05 & 0.04 \\
\hline 7 & 0.22 & 0.45 & 0.14 & 0.19 \\
\hline 8 & 0.12 & 0.13 & 0.07 & 0.04 \\
\hline 9 & 0.12 & 0.12 & 0.07 & 0.03 \\
\hline 10 & 0.12 & 0.06 & 0.08 & 0.03 \\
\hline 11 & 0.12 & 0.05 & 0.08 & 0.02 \\
\hline 12 & 0.22 & 0.30 & 0.12 & 0.05 \\
\hline 13 & 0.26 & 0.21 & 0.16 & 0.07 \\
\hline 14 & 0.44 & 0.79 & 0.24 & 0.17 \\
\hline 15 & 0.51 & 0.64 & 0.32 & 0.25 \\
\hline 16 & 0.61 & 0.35 & 0.37 & 0.15 \\
\hline 17 & 0.94 & 1.49 & 0.52 & 0.36 \\
\hline 18 & 1.78 & 1.87 & 1.00 & 0.45 \\
\hline 19 & 2.75 & 2.69 & 1.49 & 0.59 \\
\hline 20 & 3.86 & 3.38 & 2.12 & 0.74 \\
\hline 21 & 4.81 & 2.80 & 2.60 & 0.64 \\
\hline & & & & \\
\hline
\end{tabular}

*Estadísticos de 40 simulaciones. 
152 Nueva Época REMEF (The Mexican Journal of Economics and Finance)

Cuadro 3. Medias y varianzas del porcentaje de cooperación final, para diferentes umbrales de acumulación, relaciones, personas VIP, y para diferentes tasas de castigo

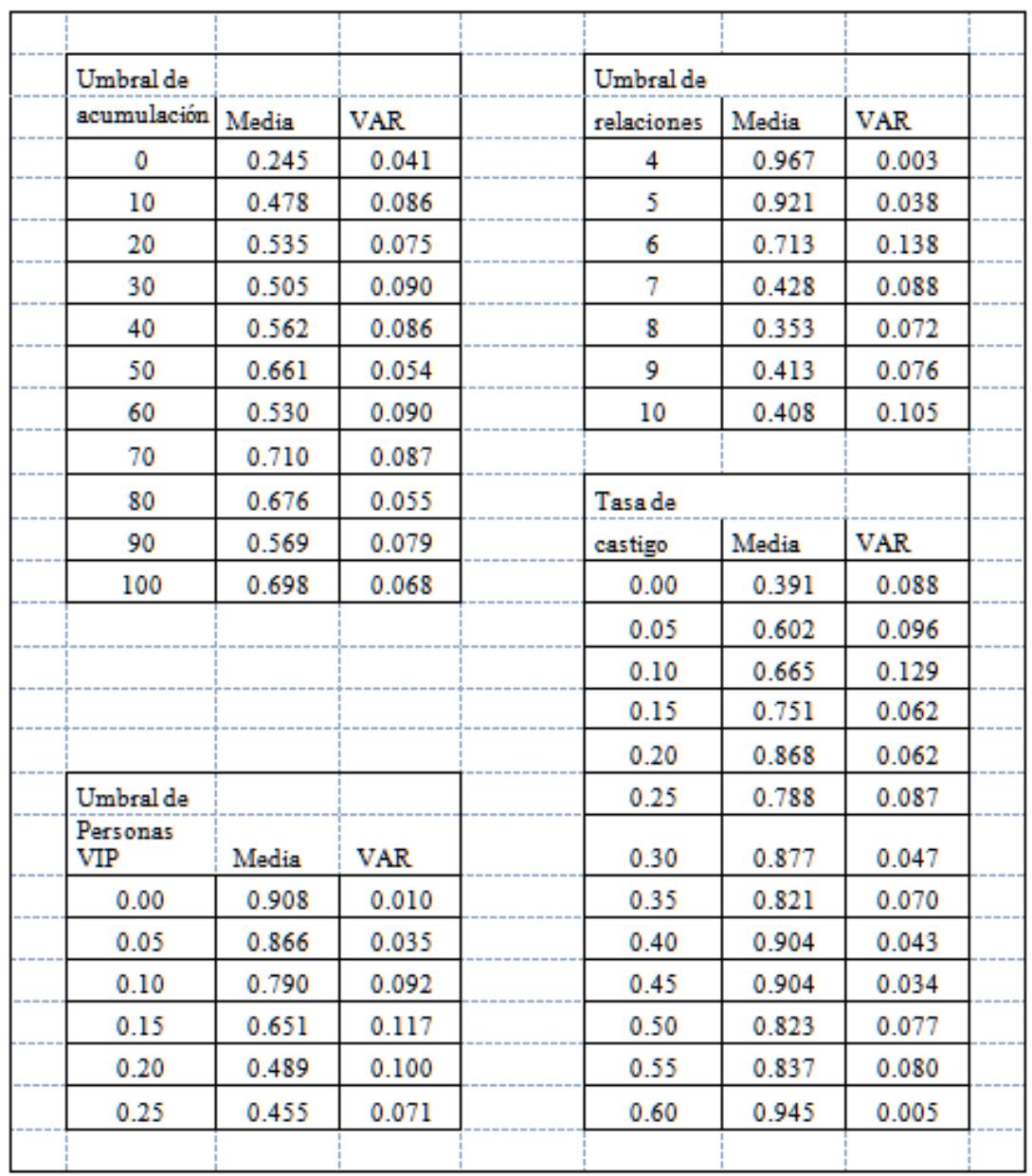

*Estadísticos de 40 simulaciones. 